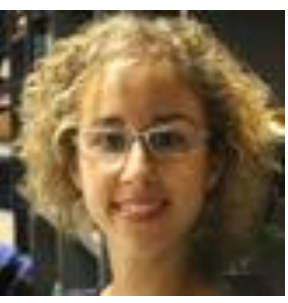

\title{
Posedición, productividad y calidad
}

Nora Aranberri

\author{
Universidad del País Vasco / Euskal Herriko Unibertsitatea
}

\section{RESUMEN}

El avance de estos últimos años en el campo de la traducción automática (TA) ha hecho posible su adopción en la industria. Y no sólo porque la calidad de los sistemas punteros haya mejorado considerablemente, sino también porque las empresas del sector han reconocido que incluso una TA imperfecta puede ser útil para satisfacer las demandas actuales del mercado de la traducción. Estamos presenciando un cambio de perspectiva con respecto al concepto de calidad. Estamos dejando atrás los estándares y modelos de evaluación que buscaban la máxima calidad en las traducciones, a favor de estándares más flexibles que se adecúan a la finalidad de los textos. Los ámbitos de uso de la traducción son múltiples, así como las expectativas de calidad de los lectores. Mientras que la traducción manual es indispensable para ciertos campos como pueden ser la medicina o el ámbito legal, donde la falta de precisión puede tener consecuencias desastrosas, o la publicidad, donde prevalece la creatividad, la TA, combinada con la posedición de mayor o menor envergadura, cubre gran parte de la demanda actual, incluso ayudando a incrementar el volumen de traducción.

Palabras clave: traducción automática, posedición, productividad, calidad.

\section{Resum (Postedició, productivitat i qualitat)}

L'avanç d'aquests últims anys en el camp de la traducció automàtica (TA) ha fet possible la seva adopció en la indústria. I no només perquè la qualitat dels sistemes punters hagi millorat considerablement, sinó també perquè les empreses del sector han reconegut que fins i tot una TA imperfecta pot ser útil per satisfer les demandes actuals del mercat de la traducció. Estem presenciant un canvi de perspectiva pel que fa al concepte de qualitat. Estem deixant enrere els estàndards i models d'avaluació que buscaven la màxima qualitat en les traduccions, a favor d'estàndards més flexibles que s'adeqüen a la finalitat dels textos. Els àmbits d'ús de la traducció són múltiples, així com les expectatives de qualitat dels lectors. Mentre que la traducció manual és indispensable per a certs camps com poden ser la medicina o l'àmbit legal, on la falta de precisió pot tenir conseqüències desastroses, o la publicitat, on preval la creativitat, la TA, combinada amb la postedició de major o menor envergadura, cobreix gran part de la demanda actual, fins i tot ajudant a incrementar el volum de traducció.

Paraules clau: traducció automàtica, postedició, productivitat, qualitat.

\section{ABSTRACT (Postediting, productivity and quality)}

This article describes the new perspective on quality as a dynamic concept, propelling the industry's adoption of machine translation, which when combined with post-editing as it usually is, offers the flexibility to meet different response times and required levels of quality. Post-editing productivity is presented as a extended model that enables companies to decide whether or not to adopt automatic translation. Information gathered is demonstrated and difficulties in implementation acknowledged. Finally, the findings of various studies that indicate that automatic translations combined with post-editing appear 
to be able to compete with traditional manual translation in terms of quality, is addressed briefly.

Keywords: Machine translation, postediting, productibity, quality.

\section{Calidad y posedición}

La TA en industria se utiliza para responder a las necesidades interlingüísticas de diversos canales de comunicación. Por una parte podemos distinguir la comunicación interna y la externa. Incluimos en comunicación interna la información multilingüe que una empresa pone a disposición de sus trabajadores bien con mera intención informativa -la empresa podría optar por no proporcionarla-, bien para el funcionamiento adecuado del trabajo información crítica para una ejecución eficaz. Aunque el nivel de exigencia se decida caso a caso, de entrada, podríamos concluir que el primer caso puede permitirse un nivel de calidad inferior que el segundo. Aun así, toda esta información permanece dentro de la empresa y, por lo tanto, se acepta un cierto margen de flexibilidad. En lo que respecta a la comunicación externa, también en este ámbito distribuye una empresa información más o menos crítica, ya sea la descripción detallada del catálogo de productos, documentación de ayuda o foros gestionados por los propios usuarios. A las condiciones impuestas por estos canales de comunicación, se deberán añadir variables que modificarán el nivel de calidad esperado, requerido y posible: la finalidad y el tipo de texto, la inmediatez y el ciclo de vida del mismo, así como el impacto que pueda tener en la imagen de la empresa (O'Brien et al., 2011).

El volumen de traducción que requiere una empresa con presencia en varios mercados o con sedes distribuidas en distintos países es abrumador y el coste inasumible en muchos casos. A esto se debería añadir la inmediatez que exigen ciertas traducciones. Dejando a un lado la inversión que esto supondría por parte de los clientes, expertos en el congreso Localization World 2013 incluso han puesto en duda la capacidad del mercado para responder a la demanda total de traducción (que cuenta con una predicción del volumen de facturación de cuarenta y siete mil millones de dólares para 2015 según el Common Sense Advisory, 2012). Sin embargo, queda claro que distintos tipos de comunicación y contextos de uso admiten distintos niveles de calidad del texto final. La TA desempeña un papel clave en esta concepción de calidad. Una vez integrado un sistema de TA dentro del flujo de trabajo de la empresa, se puede conseguir casi de manera inmediata la traducción de miles de palabras. Dependiendo del sistema, la calidad de estas traducciones será suficiente para ciertos contextos de uso. En caso de no ser así, se podrá optar por combinarla con la posedición.

Cada vez más departamentos de localización de grandes multinacionales y proveedores de servicios de traducción (PST) incorporan la TA en sus procesos de traducción. Empresas como Adobe, Autodesk, eBay, Philips y TripAdvisor, así como PSTs como Capita, Lionbridge, SDL y Welocalize han adoptado procesos de TA para la traducción de varios tipos de contenido en paralelo con la traducción manual. A veces, son las mismas empresas quienes desarrollan los sistemas de TA y traducen los textos antes de enviarlos a un PST para que complete la posedición. En otras ocasiones, son los mismos PSTs quienes, internamente o en colaboración con una empresa especializada en desarrollar sistemas de TA personalizados, utilizan la combinación de TA y posedición para hacer frente a la demanda de traducción.

La posedición puede aplicarse en distintos grados y es precisamente esta flexibilidad la que dota a la TA de capacidad de adaptación a las diferentes exigencias del mercado. Originariamente se distinguen la posedición parcial (light post-editing) y la posedición completa (full post-editing). La primera consiste en realizar los cambios necesarios e imprescindibles para que un texto pueda ser comprendido. Se hace hincapié en fenómenos que obstaculizan su lectura como pueden ser la terminología o las relaciones gramaticales incorrectas. No se trata, necesariamente, de corregir todos los errores del texto, ya que algunos errores menores pueden no alterar demasiado el texto y permitir al lector resolverlos 
por sí mismo sin detrimento para la comprensión. El texto final puede contener errores siempre y cuando éstos permitan que el mensaje se transmita satisfactoriamente. Por su parte, la posedición completa tiene como objetivo eliminar todo error de la TA y conseguir una traducción de alto nivel, a la par de la traducción tradicional manual. El texto debe ser correcto desde una perspectiva gramatical y terminológica, así como estilística, y gozar de una fluidez nativa.

Hoy en día, sin embargo, cada cliente define su propio nivel de calidad, y por consiguiente, el nivel de posedición, dando lugar a una amplia progresión de grados de posedición. Mientras que medir e implementar una calidad óptima resulta relativamente fácil, trabajar con calidades de niveles inferiores es una tarea compleja, más aún para profesionales que han sido formados para buscar la perfección en todo texto. Diversos estudios han puesto de manifiesto la necesidad de formar a especialistas en posedición dado que esta tarea requiere habilidades distintas a las que está habituado un traductor.

Así, hoy día empresas del sector como SDL, Systran o Welocalize ofrecen cursos y talleres que introducen a traductores y otros especialistas a la posedición de TA. En general, la formación consiste en ofrecer a los futuros poseditores nociones básicas sobre el desarrollo de sistemas de TA, en familiarizarlos con los distintos niveles de calidad y en mostrar, mediante actividades prácticas, errores típicos para cada par de lenguas. En la práctica industrial, para facilitar la tarea de posedición, generalmente se establece una lista de características específicas que el poseditor deberá tratar (O’Brien, 2011, Rico Pérez, 2012).

De la misma manera, la formación en posedición está empezando a llegar a las aulas de las escuelas de traducción. Poco a poco se empiezan a delinear los procesos y tareas que engloba la posedición y a definir las competencias centrales, lingüísticas e instrumentales en las que se debe instruir a los futuros poseditores (Rico y Torrejón, 2012). Emergen propuestas específicas para acercar la TA y la posedición a los alumnos y se describen las dificultades que supone la introducción y el manejo de estas tecnologías en el aula (Austermuehl, 2013; Doherty y Moorkens 2013; Doherty, Kenny y Way, 2012). Además, la formación también debe hacer frente a la mala reputación de la que goza la TA entre los traductores. Tal y como describe Arevalillo (2012), una lectura radical (y un tanto ingenua) del objetivo de la TA como tecnología que pretende sustituir a los traductores humanos ha generado una actitud defensiva y contraria a la TA entre estos profesionales. Este rechazo inicial es, en parte, culpable de una percepción distorsionada de su eficacia como herramienta de ayuda y, como resultado, de una adopción tardía y no exenta de dificultades (Gaspari et al., 2014; Teixeira, 2014).

\section{Productividad de posedición}

En vista de los beneficios que puede ofrecer la TA, cada vez más empresas se plantean introducirla en su flujo de trabajo. Uno de los modelos de evaluación para medir el impacto y la eficacia de una posible adopción de TA en industria es la productividad de posedición. Este modelo de evaluación trata de comparar la productividad de un traductor al realizar traducciones de manera tradicional 0 al poseditar una TA. Idealmente, el esfuerzo de posedición se debería medir teniendo en cuenta el esfuerzo cognitivo, el esfuerzo técnico y el esfuerzo temporal que conlleva (Krings, 2001). En general, sin embargo, los modelos que se utilizan se suelen centrar en comparar el tiempo que un poseditor o traductor necesita para completar una traducción con y sin ayuda de la TA. Asimismo, este modelo también se utiliza para comparar diferentes sistemas de TA y optar por el más conveniente para cada par de lengua, para comparar el rendimiento de un sistema con diferentes tipos de texto o productos, así como para hacer seguimientos de rendimiento tras las actualizaciones de los sistemas o cambios de poseditores.

A modo de ejemplo, veamos la implementación más extendida de este modelo en la industria. Son muchas las empresas que como Moravia, Safaba o Systran están llevando a 
cabo considerables pruebas de productividad para medir la eficiencia de sus productos y servicios. Autodesk es una de las empresas más activas en realizar (y publicar) este tipo de evaluaciones de manera regular (Plitt y Masselot, 2010; Zhechev, 2012). Con la ayuda de cuatro traductores por lengua, calculan el aumento de productividad, es decir, la diferencia de palabras traducidas en el mismo periodo de tiempo, al traducir sin ninguna herramienta de traducción asistida por ordenador (TAO) y al traducir con ayuda de la TA. Este tipo de mediciones se realiza, generalmente, en un entorno de posedición diseñado específicamente para esta tarea. En el caso de Autodesk, este entorno carece de múltiples funcionalidades que los traductores tienen a su disposición durante el proceso de traducción y, por lo tanto, no refleja con exactitud la productividad real del día a día. El uso de memorias de traducción, con su propagación continua de segmentos validados, búsquedas terminológicas en glosarios y bases de datos y accesos directos a recursos en línea, por ejemplo, acelera y enriquece el proceso traductor. En efecto, como ellos mismos reconocen y tienen planeado modificar, sus cálculos sólo tienen en cuenta el tiempo que un traductor utiliza para leer y traducir o poseditar cada segmento. No se incluye el tiempo que un traductor necesita para buscar terminología o consultar las guías de estilo, ni las pausas de descanso. Aun así, este tipo de estudios ofrecen una visión estricta de la diferencia neta entre el uso de la TA frente a la traducción manual partiendo de cero y son una buena aproximación para desvelar la capacidad con la que dotan al traductor para realizar tareas sin recursos externos. Al fin y al cabo, en una situación real, el traductor podrá acceder a recursos externos tanto en una como en la otra modalidad, con o sin TA. También resultan útiles para hacer comparativas sobre la contribución de distintos sistemas de TA.

Resulta difícil realizar una prueba de productividad que simule completamente el modo de trabajo del traductor, ya que aunque las herramientas de TAO cuentan con la posibilidad de introducir segmentos de TA, no cuentan con la opción de almacenar información sobre el tiempo utilizado para poseditar o traducir cada segmento, ni están diseñadas para registrar las operaciones exactas realizadas por el traductor durante la tarea. Incluso en casos en los que el entorno parece estar preparado, son otros factores como el perfil de los poseditores los que alejan al estudio de la realidad. Pongamos como ejemplo el estudio de Läubli et al., (2013) donde se muestra un intento de calcular la variación de productividad simulando un entorno real. Los traductores utilizan una herramienta de TAO que pone a su disposición memorias de traducción con sus correspondencias exactas y parciales, un glosario de dominio y acceso a recursos lingüísticos externos, ya sean en línea o en papel. La tarea de posedición añade la TA para los segmentos que carecen de correspondencias de la memoria. Los traductores, sin embargo, son estudiantes de grado y posgrado con mínima experiencia traductora. Aunque podamos comparar la productividad de profesionales al comienzo de sus carreras, no aportan datos sobre la realidad de un entorno laboral activo.

Diversos estudios de investigación y casos de uso revelan un aumento de la productividad gracias a la TA. En su última evaluación, Autodesk informó sobre una franja de incremento que varía desde un 37\% para el polaco, hasta un 92\% para el francés (Zhechev, 2012). Mientras que el rendimiento medio de un traductor se suele establecer en unas 2000 palabras por día, la posedición parece aumentar esta media a 3 000-9 000 palabras por día. Ahondando un poco más, se observa que el incremento en productividad varía considerablemente en función del par de lenguas, el texto y el poseditor que completa la tarea. Vemos, por tanto, que la productividad está marcada por la calidad de la traducción automática, así como por la especialización de los poseditores.

\section{Calidad de posedición}

Casi intuitivamente, un poseditor mantiene la traducción original en la medida de lo posible dependiendo de la calidad de la traducción proporcionada por el sistema de TA, mientras que inserta cambios para que el texto se ajuste a los estándares de calidad marcados por el cliente. De algún modo, se podría decir que el poseditor busca la manera de transformar el candidato de traducción con el número mínimo de cambios posible. Esta 
naturaleza de la posedición arroja dudas sobre la calidad final de la traducción -hablamos aquí de una posedición completa. Reducir el tiempo de traducción sólo es rentable si la calidad final de dicha traducción es la esperada y la que se conseguiría siguiendo el modelo de traducción sin TA.

Mientras algunos detractores de la TA argumentan que los textos poseditados son rígidos y pierden fluidez con respecto a las traducciones manuales, diversos estudios han demostrado lo contrario (O'Curran, 2014). Tras mostrar traducciones obtenidas mediante uno y otro método, los evaluadores parecen no ser capaces de distinguir entre traducciones manuales y traducciones obtenidas mediante la combinación de TA y posedición (Carl et al., 2011; Läubli et al., 2013). Es más, en ocasiones se puntúa más favorablemente a las traducciones resultantes de TA con posedición. Damos por sentado que la traducción manual y validada tras un proceso de revisión es siempre correcta pero análisis comparativos como el de Gueberof (2009) demuestran que no siempre es así. Su estudio reveló que más de la mitad de los errores registrados durante el proceso de análisis de errores de traducciones manuales y automáticas con posedición provenían de segmentos ya validados de la memoria de traducción (MT). Tal y como apunta la autora, la explicación puede radicar en que los errores encontrados en la MT son errores de traducción y no lingüísticos, y por lo tanto, son difíciles de detectar sin volver al segmento de la lengua original. Sin embargo, los errores que encontramos en las TA son obvios y fáciles de identificar. Otro punto a favor de la TA es que favorece la homogeneidad léxica y terminológica, e incluso fraseológica. La rigidez o falta de variación lingüística que a veces se le achaca, ocasionada bien por la limitación de los diccionarios bilingües o por la ausencia de alternativas en los corpus de entrenamiento, resulta en una selección determinista de las equivalencias a lo largo del texto.

Como hemos dicho, la calidad de la posedición estará supeditada a la calidad de traducción que genere el sistema de TA y a la capacidad del poseditor para transformar dicha TA en un texto correcto y fluido. Aún así, en general, experiencias industriales como la de Olga Loskutova, especialista de TA en Paypal, muestran que la TA, además de reducir los costes y el tiempo de traducción, ayuda a mejorar la calidad.

Para concluir, merece destacar un nuevo contexto de uso de la TA combinada con la posedición que ha nacido gracias a la popularización de la TA en los últimos años: posedición de TA para usuarios no profesionales. No son solamente las empresas quienes ven la TA como una oportunidad para mejorar y acelerar el proceso de traducción. Usuarios que debido a la naturaleza de su trabajo se ven envueltos en tareas de traducción esporádicamente están empezando a interesarse en la TA (Laurenzi et al., 2013). Y a raíz de este interés, están surgiendo plataformas especializadas como puede ser el Bologna Translation Service dirigido específicamente a estos grupos. Se trata de entornos de trabajo sencillos que generalmente incluyen la opción de combinar memorias de traducción con traducciones automáticas, y que carecen del abanico de funcionalidades que se ofertan a los traductores profesionales, ya que no resultan tan relevantes para estos usuarios y complican la adopción y uso del entorno. En ocasiones es el propio usuario quien decide la calidad necesaria de los textos, pues es él mismo quien los utilizará y divulgará posteriormente. Los usuarios consideran la TA una herramienta que acelera la producción del texto final y, en general, parecen tener una actitud más benevolente hacia la TA. Un estudio reciente (Aranberri et al., 2014) apunta a que incluso con una TA de calidad discutible aumenta la productividad de usuarios no profesionales. Gracias al conocimiento del campo temático de los contenidos, estos usuarios aprovechan las sugerencias de la TA al máximo, discriminando fácilmente entre propuestas correctas y erróneas, y evitan, en gran parte, la tarea de búsqueda terminológica, así como el síndrome de la página en blanco.

\section{Conclusión}

A lo largo de este artículo hemos abordado la nueva perspectiva de calidad, que ha pasado a ser una noción dinámica, en contraste con la concepción estática de perfección con 
la que se imponía anteriormente. Así, hemos visto que la traducción automática, generalmente combinada con la posedición, ha pasado a tener una posición central dentro de la industria de la traducción, gracias a la flexibilidad que ofrece para satisfacer los diferentes niveles de calidad y tiempo de respuesta. La productividad de posedición ha emergido como modelo extendido para decidir si una empresa debería adoptar o no la traducción automática. A pesar de la dificultad de simular entornos reales para este tipo de modelo de evaluación, hemos visto que la mayoría de estudios corroboran el aumento del rendimiento gracias a la TA, aunque éste varíe en función de la calidad de traducción del sistema y la especialización de los poseditores. Finalmente, hemos tratado la calidad de las traducciones automáticas combinadas con posedición, que según estudios, parece poder competir con la traducción manual tradicional.

\section{Bibliografía}

Aranberri, N., Labaka, G., Diaz de llarraza, A. y Sarasola, K. (2014). "Comparison of postediting productivity between professional translators and lay users", en Proceeding of AMTA Third Workshop on Post-editing Technology and Practice (WPTP-3), Vancouver, Canada, 26 October 2014, páginas 20-33.

Arevalillo, J. J. (2012). “La traducción automática en las empresas de traducción”. Tradumàtica, 10, páginas 179-184.

Austermuehl, F. (2013). "Future (and not-so-future) trends in the teaching of translation technology”, Tradumàtica, 11, páginas 326-337.

Carl, M., Dragsted, B., Elming, J.. Hardt, D. y Lykke Jakobsen, A. (2011). "The process of post-editing: a pilot study", Sharp, Bernadette, Michael Zock, Michael Carl and Arnt Lykke Jakobsen (eds), en Proceedings of the 8th international NLPSC workshop. Special theme: Human-machine interaction in translation, páginas 131-142, Copenhagen Business School, Copenhagen, Denmark, 20-21 August 2011. Frederiksberg: Samfundslitteratur.

Common Sense Advisory. Kelly, N., DePalma, D. and Steward, R. G. (2012). The Language Services Market: 2012.

Doherty, S. y Moorkens, J. (2013). "Investigating the experience of translation technology labs: pedagogical implications”. The Journal of Specialised Translation 19:122-136.

Doherty, S., Kenny, D. and Way, A. (2012). "Taking statistical machine translation to the student translator", en Tenth Conference of the Association for Machine Translation in the Americas, San Diego, California.

Gaspari, F., Toral, A., Naskar, S., Groves, D. and Way, A. (2014). "Perception vs Reality: Measuring Translation Post-Editing Productivity", en Proceeding of AMTA Third Workshop on Post-editing Technology and Practice (WPTP-3), Vancouver, Canada, 26 October 2014, páginas 60-72.

Gueberof, A. (2009). "Productivity and quality in MT post-editing”, en Proceedings of the MT Summit XII Workshop Beyond Translation Memories: New Tools for Translators MT, Ottawa, Ontario, Canada.

Krings, H. P. (2001). Repairing Texts: Empirical Investigations of Machine Translation PostEditing Processes. Kent, Ohio: The Kent State University Press. Edited/translated by G.S. Koby.

Läubli, S., Fishel, M., Massey, G., Ehrensberger-Dow, M. y Volk, M. (2013). “Assessing PostEditing Efficiency in a Realistic Translation Environment” en Sharon O'Brien, Michel Simard y Lucia Specia (eds.) en Proceedings of MT Summit XIV Workshop on Postediting Technology and Practice, Nice, September 2, 2013, páginas 83-91. 
Laurenzi, A. Brownstein, M., Turner, A. M., y Kirchhoff, K. (2013). "Integrated Post-Editing and Translation Management for Lay User Communities", en Proceedings of MT Summit Workshop on Post-editing Technology and Practice, Nice, France, 2 September 2013.

O'Brien, S. (2011). "Introduction to Post-editing: Who, What, How and Where to Next?" en Tutorial at the ninth Conference of the Association for Machine Translation in the Americas, 31 October 2010.

O'Brien, S., Choudhury, R., van der Meer, J. y Aranberri, N. (2011). "Translation Quality Evaluation Framework". TAUS Report.

O'Curran, E. (2014). "Translation Quality in Post-Edited versus Human-Translated Segments: A Case Study", en Proceeding of AMTA Third Workshop on Post-editing Technology and Practice (WPTP-3), Vancouver, Canada, 26 October 2014, páginas 113-118.

Plitt, M., y Masselot, F. (2010). "A productivity test of statistical machine translation postediting in a typical localisation context", The Prague Bulletin of Mathematical Linguistics 93:7-16.

Rico Perez, C. (2012). "A Flexible Decision Tool for Implementing Post-editing Guidelines." Localization Focus, The International Journal of Localization, 11(1), Localisation Research Centre.

Rico, C. y Torrejón, E. (2012). "Skills and Profile of the New Role of the Translator as MT Post-editor", Tradumàtica, 10, páginas 166-178.

Teixeira, C. (2014). "Perceived vs. measured performance in the post-editing of suggestions from machine translation and translation memories", en Proceeding of AMTA Third Workshop on Post-editing Technology and Practice (WPTP-3), Vancouver, Canada, 26 October 2014, páginas 45-59.

Zhechev, V. (2012). "Machine Translation Infrastructure and Post-editing Performance at Autodesk", en AMTA 2012 Workshop on Post-Editing Technology and Practice (WPTP 2012), páginas 87-96, San Diego, USA, October. Association for Machine Translation in the Americas (AMTA). 